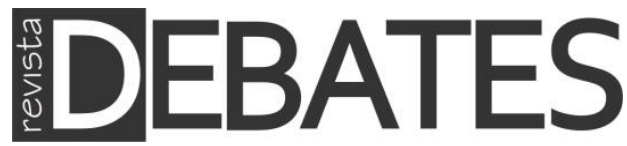

\title{
Congruencia temática entre ciudadanos y representantes en América Latina: una perspectiva multidimensional
}

\author{
Issue congruence between citizens and representatives in Latin \\ America: a multidimensional perspective
}

\section{Juan Antonio Rodríguez-Zepeda}

\section{Resumen}

Este trabajo propone una contribución metodológica al estudio empírico de la representación política. En él se explora el nivel de congruencia temática entre los ciudadanos y los diputados de 15 países latinoamericanos. El objetivo es conocer en qué medida concuerdan las opiniones de estos dos grupos con respecto a la intervención del Estado en seis aspectos del ámbito público. Para dicho propósito se utilizaron dos métodos de estadística multivariante: el escalamiento multidensional y el análisis Procrustes. Por medio del primero fue posible saber cómo están estructurados estos temas para representantes y representados; a partir del segundo se midió el grado de semejanza entre dichas estructuras. La contribución del estudio es considerar la estructura multidimensional de la congruencia temática y usar herramientas estadísticas adecuadas para analizarla.

\section{Palabras clave}

Congruencia Temática; América Latina; Análisis Multivariante.

\begin{abstract}
This paper proposes a methodological contribution to the empirical study of political representation. It explores the level of issue congruence between citizens and legislators from 15 Latin American countries. The goal is find out the degree of agreement between the attitudes of these two groups with respect to the intervention of the State in six aspects of the public sphere. For this purpose, two multivariate statistical methods were used: multidimensional scaling and Procrustes analysis. Through the first it was possible to know how these issues are structured for representatives and citizens; the second one provided an assessment of the degree of similarity between these structures. The contribution of the study is to consider the multidimensionality of issue congruence and the use of appropriate statistical tools to analyze it.
\end{abstract}

\section{Keywords}

Issue Congruence; Latin America; Multivariate Analysis. 


\section{Introducción ${ }^{1}$}

¿Están los representantes latinoamericanos cercanos a las actitudes y demandas de los ciudadanos? Esta es una pregunta que en años recientes ha protagonizado numerosos estudios sobre la representación política desde un punto de vista empírico. Así, son muchos los análisis que se han enfocado en el grado en que las preferencias de los ciudadanos y sus representantes coinciden, o qué tanto las políticas públicas implementadas por estos últimos responden a las demandas de los primeros. Dichos trabajos se han llevado a cabo denominando este fenómeno de distintas formas - congruencia, correspondencia, vínculos o responsiveness (receptividad) - y aproximándose a él a través de diferentes enfoques metodológicos.

Pese a la atención que ha despertado este tema en democracias consolidadas, los estudios sobre congruencia ideológica o temática enfocados en América Latina todavía son escasos. Las causas principales de esta situación son los recientes procesos de consolidación democrática, los recurrentes periodos de inestabilidad partidista, la fluidez en la oferta de partidos y, especialmente, la ausencia de información sistemática y comparable sobre ciudadanos y élites políticas. Los textos de Luna y Zechmeister (2005), Otero-Felipe y Rodríguez-Zepeda (2010 y 2014), Saiegh (2015), Lupu et al. (2017) y Otero-Felipe (2017) constituyen algunos de los ejemplos de este tipo de análisis comparados centrados en la región.

En este sentido, hay dos elementos comunes en esta literatura. Por un lado, la aproximación metodológica al estudio de la congruencia, centrándose sobre todo en su operacionalización. La mayoría de trabajos han medido la congruencia como una distancia, ya sea entre las medias de las posiciones de representados y representantes, o la distancia de las posiciones individuales a la posición media de uno de los grupos. Recientemente se ha usado la comparación entre las distribuciones estadísticas de dichas posiciones, pero generalmente haciendo comparaciones tema a tema, sin sacar partido a la estructura multivariante de este fenómeno multidimensional ${ }^{2}$. En segundo lugar, ha habido una tendencia mayoritaria hacia el estudio de la congruencia ideológica frente al análisis de cuestiones políticas concretas. En contextos como el europeo, donde la izquierda y la derecha estructuran en gran medida los temas políticos, los estudios que han comparado el grado de conexión

\footnotetext{
${ }^{1}$ Este trabajo se ha realizado con la financiación del Proyecto "Competición ideológica y sistema político: escenarios de estabilidad y escenarios de cambio” CSO2015-63555-R (MINECO/FEDER), dirigido por Leticia M. Ruiz.

${ }^{2}$ El término multivariante se refiere a la consideración en el análisis de dos o más indicadores (variables) que están variando a la vez.
} 
entre partidos y votantes o ciudadanos y gobierno en términos ideológicos han sido tomados como un indicador indiscutible de la existencia de representación política.

Aunque se asume que la dimensionalidad y la composición de los conceptos de izquierda y derecha pueden variar en términos contextuales, ésta sigue siendo la dimensión predominante y el atajo informativo más utilizado tanto por los ciudadanos como por los partidos políticos. Sin embargo, las dudas persisten en contextos como el latinoamericano donde la ideología puede tener un menor peso en la estructuración de las preferencias ciudadanas y de las élites políticas. Por lo tanto, es pertinente enfocar el estudio de la congruencia desde los temas relevantes del debate político, sin olvidar que estos están relacionados con la dimensión ideológica.

Este trabajo constituye una contribución al estudio de la representación política empírica en América Latina en estos dos aspectos. En primer lugar, se presenta una aproximación metodológica novedosa al estudio de la congruencia; ésta supone superar algunas de las limitaciones de estudios anteriores que han abordado el tema de la congruencia basándose en un solo indicador o comparando las posiciones de varios indicadores de forma individual. Para llevarlo a cabo se utilizaron dos métodos de estadística multivariante que no han sido aplicados juntos en el análisis de la congruencia: el Escalamiento Multidimensional y el análisis Procrustes ${ }^{3}$. El primer método permite conocer cómo están estructurados estos temas para los ciudadanos y para los diputados; mientras que el segundo mide el grado de semejanza entre dichas estructuras, ofreciendo una perspectiva mucho más completa de este fenómeno. Además, se explora el nivel de congruencia temática entre ciudadanos y los miembros de la cámara baja (diputados) del Congreso en quince países latinoamericanos. Así, el objetivo de este estudio fue conocer en qué medida eran concordantes las opiniones de estos dos grupos con respecto a la intervención del Estado en la economía, un tema que si bien es próximo a la dimensión ideológica, presenta características singulares en cada sistema político (GRAMACHO y LLAMAZARES, 2007).

A partir del análisis llevado a cabo, se determinó en qué medida los representantes coinciden con los ciudadanos en sus consideraciones sobre el papel de intervención del Estado. Los resultados obtenidos muestran que hay diferencias tanto entre los países estudiados, como en el grado de concordancia en los temas analizados.

\footnotetext{
${ }^{3}$ En Luna (2014) se usa el análisis Procrustes, pero no se parte de las configuraciones producidas por el Escalamiento Multidimensional.
} 
Se presenta así nueva evidencia empírica sobre el grado de congruencia temática en la región. Esto nos permite contrastar los hallazgos con estudios previos y plantear hasta qué punto una mayor o menor correspondencia entre las opiniones de ciudadanos y sus representantes constituye un termómetro de la capacidad de respuesta de los gobernantes a las demandas de los gobernados y, por lo tanto, puede ser un indicador adecuado de la calidad de la democracia.

El presente artículo tiene seis secciones. A continuación se discuten brevemente las aportaciones teóricas y metodológicas más destacadas de la literatura sobre el estudio de la congruencia ideológica y temática. La siguiente sección presenta los estudios comparativos sobre estos vínculos en Latinoamerica. Posteriormente se describen detalladamente los datos utilizados en el análisis empírico. La cuarta sección expone los métodos de estadística multivariante empleados para operacionalizar la congruencia. Después se comentan los resultados obtenidos al aplicar dicha metodología en quince países de la región. La última sección presenta las conclusiones e implicaciones del estudio.

\section{Congruencia ideológica y temática como un indicador de la representación}

La evaluación de la representación política de las democracias es una de las cuestiones que más debates ha generado en la disciplina, tanto desde un punto de vista teórico como empírico. Powell (2004) diferenció dos aproximaciones relacionadas que han recibido una desigual atención en la literatura. La primera, que aborda la representación procedimental, analiza cómo las elecciones vinculan a ciudadanos y representantes a partir de la traducción de los votos y escaños. El segundo enfoque, de tipo sustantivo, examina en qué medida ciudadanos y representantes están conectados en una serie de cuestiones políticas. Bajo esta perspectiva empírica del estudio de la representación política podemos encontrar el issue congruence, la cual estudia la congruencia o correspondencia de actitudes entre representantes y representados en un sistema político.

Los primeros análisis de la congruencia se acercaron a este fenómeno de un modo más descriptivo, mostrando desde diferentes métodos cómo analizar y evaluar el grado de coincidencia entre las preferencias de los ciudadanos - ya sea con respecto a cuestiones políticas de diversa naturaleza o al tipo de políticas públicas susceptibles de ser aplicadas $-\mathrm{y}$ las posiciones que tienen sus representantes al respecto (MILLER y STOKES, 1963; ACHEN, 1977 y 1978; WEISSBERG, 1978; DALTON, 1985; CONVERSE y PIERCE, 1986). Estudios comparados posteriores se encargaron no 
sólo de describir el nivel de congruencia, sino también de señalar los elementos explicativos del grado en que legisladores, partidos o gobiernos y los ciudadanos (o electorados) están coordinados en sus preferencias ideológicas o hacia una serie de cuestiones políticas (DALTON, 1985; THOMASSEN y SCHMITT, 1999; LUNA y ZECHMEISTER, 2005; BLAIS y BODET, 2006; GOLDER y STRAMSKI, 2010; OTERO-FELIPE, 2017). Así, estos trabajos han destacado la relevancia que para la calidad de la democracia tiene la existencia de vínculos ideológicos y programáticos. Ahora contamos con un amplio cuerpo teórico y metodológico para analizar este fenómeno, de modo que podemos enfatizar cuáles son los aspectos relevantes que hay que tener en cuenta por sus implicaciones en los resultados: el enfoque adoptado, las dimensiones o temas analizados y los indicadores utilizados para medir la congruencia.

Respecto al primer elemento, esta literatura ha tomado dos enfoques diferentes, uno de tipo colectivo - "collective" representation -, que implica el estudio de los niveles de correspondencia entre el conjunto del órgano representativo y los ciudadanos representados en él, o la congruencia entre los ciudadanos y el gobierno. El segundo aborda la conexión diádica - "dyadic" representation considerando legisladores o partidos frente a sus votantes (WEISSBERG, 1978). Aunque son complementarios, ambos enfoques tienen una contribución diferente al estudio de la representación. El primero presenta una perspectiva amplia y sistémica de la congruencia, y el segundo tiene una mayor conexión con el ámbito partidista y electoral.

En relación a las cuestiones analizadas, la literatura ha mostrado también mucha variedad, aunque en este caso la disponibilidad de evidencia empírica explica en gran medida estas diferencias. Sobre los Estados Unidos encontramos análisis de congruencia enfocados en diversos temas, desde políticas públicas concretas a objetos de estudio más laxos referentes a grado de liberalismo o conservadurismo existente en las élites políticas y los ciudadanos (BURSTEIN, 2003). En los países de Europa occidental, la creciente disponibilidad de encuestas de opinión pública ha hecho posible realizar nuevos estudios de vínculos entre representantes y ciudadanos sobre cuestiones políticas diferentes de la ideología. Esta situación ha permitido una mayor variedad de trabajos donde se analizan tanto cuestiones estructurales, vinculados a las clásicas dimensiones de la competencia izquierda y derecha, como temas no estructurales. 
El tercero de los elementos, y tal vez el más relevante por su influencia en los resultados, se refiere a los indicadores utilizados para medir la congruencia ${ }^{4}$. El trabajo de Miller y Stokes (1963) fue también pionero en establecer la medición de vínculos entre representantes y representados, usando como indicador las correlaciones entre opiniones de los electorados y las de los legisladores. Así, un valor más alto del coeficiente de Pearson corresponde a mayor congruencia. Frente a este indicador, podemos encontrar una medida más simple y muy utilizada que es el cálculo de las distancias entre actores, bien utilizando la posición media (o mediana) del partido y la posición de los votantes de ese partido, bien la distancia entre el ciudadano mediano y el gobierno o legislador mediano5.

Las críticas a este tipo de aproximaciones no tardaron en aparecer al no considerar el efecto que la distribución de las opiniones podía tener en los hallazgos. En esta línea Achen (1977 y 1978) propuso tres indicadores para medir diferentes dimensiones de la representación política, siendo las dos primeras una medición de distancias más elaboradas: la proximidad, que se refiere a la convergencia de posturas entre el partido y cada uno de los votantes; el centrismo, que mide el grado de acuerdo absoluto entre un partido y el promedio de los votantes, y la receptividad, que estima el acuerdo relativo entre los partidos y la media de los votantes. En cualquiera de ellas una menor distancia indica una mayor congruencia entre los representantes y representados.

Siguiendo la metodología iniciada por Achen (1978), Golder y Stramski (2010) proponen una medición de la congruencia de tipo colectivo, a través de la comparación de la distribución de preferencias de ciudadanos y representantes conocida como "many to many". Por su parte, Saiegh (2015) usó métodos de escalamiento conjunto para posicionar a ciudadanos y legisladores en una escala ideológica común. Estas aproximaciones tienen como elemento común que no exploran el carácter multidimensional de la congruencia. Un avance importante en esa dirección ha sido elaborado por Lupu et al. (2017). Estos autores proponen usar una medida llamada Earth Mover's Distance para medir la congruencia temática desde una perspectiva multidimensional ${ }^{6}$.

\footnotetext{
${ }^{4}$ Para una completa revisión de las medidas de congruencia ver Otero-Felipe (2017).

${ }^{5}$ Una variante de ésta es la sustracción de la media de la postura de los votantes de la que exhiben los partidos, elevándolo posteriormente al cuadrado, para crear la distancia Euclidiana.

${ }^{6}$ Dicho artículo presenta un sólido argumento sobre las bondades de esta medida. Sin embargo, hay dos elementos que hacen más atractivo el enfoque presentado aquí. Primero, como se mostrará más adelante, al hacer un análisis por pasos es posible saber qué temas generan variaciones en los niveles de
} 


\section{El estudio de la congruencia ideológica y temática en América Latina}

La literatura sobre este tema enfocada en América Latina ha sido muy escasa, al compararla con otras regiones, situación que puede explicarse por dos razones. En primer lugar se debe a la disponibilidad de evidencia empírica adecuada para llevar a cabo estos análisis porque, aunque se cuenta con encuestas de opinión pública desde mediados de los años noventa a partir de las cuales extraer las posiciones y actitudes de la ciudadanía, ha sido más complejo contar con datos coincidentes en el tiempo sobre los representantes. La segunda razón tiene que ver con una inercia, actualmente menos favorecida en la literatura, que presentaba la política latinoamericana como predominantemente personalista, clientelar y populista, donde los elementos programáticos e ideológicos eran minoritarios. Con la consolidación democrática, los parlamentos y los partidos políticos han ido ocupando un papel cada vez más preponderante en los estudios politológicos de la región, y con ello ha aumentado la preocupación en torno a su tradicionalmente discutida estructuración ideológica y en particular cómo han construido estos vínculos con los ciudadanos.

En este sentido, uno de los primeros estudios comparado sobre el grado de congruencia en la región es el de Luna y Zechmeister (2005). En él los autores mostraron, a nivel agregado, los diferentes niveles de vinculación temática y las variables que estaban relacionadas con la congruencia. Sin embargo, buena parte de la literatura sobre vínculos ideológicos y programáticos en la región se compone de estudios centrados en un solo país. Por otro lado, recientemente se han desarrollado estudios comparados donde se analiza bajo diferentes enfoques metodológicos el tipo de vinculación entre representantes y representados en la región (p. ej. OTEROFELIPE y RODRÍGUEZ-ZEPEDA, 2010; SAIEGH, 2015; LUPU et al. 2017; OTERO-FELIPE, 2017).

La mayoría de estos trabajos se enfocan en la ideología, y no en temas políticos concretos, como parámetro para evaluar el grado de congruencia. Esto puede suponer una limitación al simplificar la realidad política a una única dimensión. Hay que tener en cuenta, además, que el propio contenido de las etiquetas izquierda y derecha y su conexión con temáticas particulares puede ser muy diferente tanto para las élites políticas o los partidos como para ciudadanos (ZECHMEISTER, 2006; SAIEGH, 2015). Así, el análisis de temas políticos que se

congruencia. Segundo, los autores no discuten el efecto que genera en su medida el usar ítems (variables) que tienen diferentes escalas de medición; este problema no está presenta aquí. 
lleva a cabo en el presente artículo deja al margen la tradicional aproximación ideológica y se centra en seis temas políticos, referentes a la intervención del Estado. Una aproximación que supone un paso más en el estudio de la congruencia en la región, y que permite acumular y contrastar los hallazgos obtenidos en trabajos anteriores.

El segundo elemento destacado de los estudios de la congruencia en la región es de tipo metodológico, porque en buena medida reproducen mediciones aplicadas en otras regiones, sean correlaciones, distancias o distribuciones de frecuencias. Así, no sorprende que existan resultados heterogéneos en torno a qué sistemas o partidos políticos resultan más representativos. Por lo tanto, es necesario proponer métodos basados en técnicas estadísticas adecuadas para analizar la congruencia temática considerando su carácter multidimensional.

Es en esta cuestión donde el presente trabajo tiene su principal contribución al estudio de la congruencia. Aquí se utilizan dos técnicas estadísticas para aproximarse a la medición de la congruencia en varios países de la región desde perspectivas hasta ahora no utilizadas. En primer lugar, se usa el Escalamiento Multidimensional que permite estudiar las estructuras de estos datos a partir de una reducción de su dimensionalidad, y hacer una primera valoración de las semejanzas, o diferencias, entre las actitudes de los representantes y representados. Por su parte, el análisis Procrustes es una herramienta cuyo propósito es la comparación de forma paramétrica de dos configuraciones de puntos, que en este caso son las coordenadas obtenidas en un análisis previo. Así, este estudio se circunscribe en esta línea de investigación bajo la perspectiva de la collective representation, ya que se compararán las posiciones de todos los legisladores miembros de la cámara de diputados con las posiciones de todos los ciudadanos en cada país incluido en el estudio.

\section{Descripción de casos y evidencia empirica}

Como se especificó anteriormente, en este trabajo se lleva a cabo una aproximación a la congruencia temática de tipo colectivo a partir de dos técnicas de análisis que no han sido utilizadas conjuntamente en tipo de estudios. De este modo se aparta de la predisposición que existe en la literatura a recurrir a las distancias o a la comparación de distribuciones de frecuencias, pero también a utilizar la dimensión ideológica como el único indicador del grado de vinculación entre ciudadanos y representantes en la región.

En relación a los temas objeto de análisis, se prefirió escoger aquellos que estuvieran en el debate público, y que generaran politización o controversias entre la 
ciudadanía y los actores políticos. Además, se privilegiaron temas que fueran influyentes en la competencia electoral y que tuvieran una gran estabilidad en la agenda pública de la región. En este sentido, el papel del Estado en la economía es probablemente la cuestión que mejor se ajusta a dichas características; a pesar de su conexión con la dimensión izquierda-derecha, mantiene una singularidad en función de los contextos nacionales (GRAMACHO y LLAMAZARES, 2007). Es pertinente recordar además que en América Latina se vivió durante la década de los años ochenta una etapa de profundas reformas económicas que tuvieron un gran impacto en la actuación del Estado en los países no sólo en el ámbito económico sino también en el político, dando un vuelco a las políticas intervencionistas de las décadas anteriores (STOKES, 2001). Sin embargo, durante los últimos años el éxito electoral de varios partidos de izquierda, que ocuparon los gobiernos nacionales en gran parte de los países de la región, hizo resurgir el debate sobre estas cuestiones, recuperando una agenda pública nuevamente intervencionista en varios países (FLORES MACÍAS, 2012). Por todas estas razones, se consideró pertinente analizar el grado en que ciudadanos y representantes coincidían en valorar cuestiones relativas al papel del Estado y su grado de intervención en los ámbitos económico y social.

Para el estudio de la congruencia en torno a esta temática se han utilizado datos de encuestas de opinión pública y de élites parlamentarias. Los datos de opinión pública provienen del Proyecto de Opinión Pública de América Latina (LAPOP) disponibles para el año 20107. Por su parte, los datos utilizados para conocer las posiciones de los diputados (legisladores) provienen del proyecto Élites Parlamentarias en América Latina (PELA), basado en la aplicación de cuestionarios estructurados y uniformes en los diferentes países latinoamericanos ${ }^{8}$. El uso de estas encuestas a los representantes tiene además ventajas claras sobre el uso de otro tipo de evidencia empírica que en ocasiones se ha utilizado en los estudios de congruencia, como pueden ser el uso de bases de expertos (CASTLES y MAIR, 1984), la información obtenida de los programas y documentos partidarios (BUDGE et al., 2001), o la extracción de las posiciones y actitudes a partir de las opiniones que señalan los ciudadanos (GOLDER y STRAMSKI, 2010) y que pueden generar problemas de endogeneidad y validez en los resultados. Además, hay dos ventajas

\footnotetext{
7 Agradezco al Proyecto de Opinión Pública de América Latina (LAPOP) por hacer accesibles estas bases de datos: www.LapopSurveys.org.

${ }^{8}$ Agradezco la disponibilidad de los datos del Proyecto de Élites Parlamentarias Latinoamericanas (PELA): http://americo.usal.es/oir/elites/bases_de_datos.htm.
} 
añadidas. Una es el uso de cuestiones que tienen exactamente la misma redacción en ambas bases de datos, con lo cual se evitan posibles problemas que son comunes en otros trabajos y que pueden afectar a la validez de los resultados (HERRERA et al., 1992). La segunda es la disponibilidad de datos para realizar comparación entre un gran número de países, porque ambos proyectos aplican cuestionarios comunes en todos los casos, posibilitando la comparación de los resultados entre países.

El análisis empírico se llevó a cabo a partir de los datos disponibles para 15 países de América Latina9. Todas las encuestas a ciudadanos (LAPOP) se realizaron durante el ańo $2010^{10}$. Por su parte, las encuestas a los diputados (PELA) suelen llevarse a cabo en la primera mitad de legislatura, dependiendo de la fecha de realización de las elecciones. Las encuestas a ciudadanos fueron emparejadas con los sondeos a diputados de la legislatura que les corresponden. A continuación, se presentan las preguntas tal y como fueron aplicadas a los ciudadanos y los diputados de los países estudiados ${ }^{11}$. También se incluye la palabra con la que serán referidos dichos temas en los análisis estadísticos realizados.

\footnotetext{
${ }^{9}$ Los países incluidos en el análisis son: Argentina, Bolivia, Brasil, Chile, Colombia, Costa Rica, Ecuador, El Salvador, Guatemala, Honduras, México, Nicaragua, Perú, República Dominicana y Uruguay.

${ }^{10}$ La razón para seleccionar sólo este año es que fue la única ronda de las encuestas LAPOP donde se incluyó completa la batería de preguntas sobre la intervención Estatal.

${ }^{11}$ La redacción de las preguntas, y la escala de las respuestas, fue exactamente igual para ambos grupos de personas. Esta situación elimina dudas sobre la igualdad de la interpretación de las preguntas encontrados en otros estudios donde ciudadanos y representantes respondieron preguntas similares, pero no idénticas. Además, el tener escalas iguales también hace comparables los resultados de los análisis estadísticos.
} 
Cuadro 1 - Temas incluidos en el análisis

\begin{tabular}{|c|c|}
\hline $\begin{array}{l}\text { Encabezado } \\
\text { común a todas } \\
\text { las preguntas }\end{array}$ & $\begin{array}{l}\text { Ahora le voy a leer unas frases sobre el rol del Estado. Por favor, dígame hasta qué } \\
\text { punto está de acuerdo o en desacuerdo con ellas. Para ello utilice la siguiente } \\
\text { escala de } 1 \text { a } 7 \text {, donde el " } 1 \text { " significa muy en desacuerdo y el " } 7 \text { " muy de } \\
\text { acuerdo. }\end{array}$ \\
\hline Empresas & $\begin{array}{l}\text { El Estado X, en lugar del sector privado, debería ser el dueńo de las empresas e } \\
\text { industrias más importantes del país. ¿Hasta qué punto está de acuerdo o en } \\
\text { desacuerdo con esta frase? }\end{array}$ \\
\hline Bienestar & $\begin{array}{l}\text { El Estado X, más que los individuos, debería ser el principal responsable de } \\
\text { asegurar el bienestar de la gente. ¿Hasta qué punto está de acuerdo o en } \\
\text { desacuerdo con esta frase? }\end{array}$ \\
\hline Empleos & $\begin{array}{l}\text { El Estado X, más que la empresa privada, debería ser el principal responsable de } \\
\text { crear empleos. ¿Hasta qué punto está de acuerdo o en desacuerdo con esta frase? }\end{array}$ \\
\hline Desigualdad & $\begin{array}{l}\text { El Estado X debe implementar políticas firmes para reducir la desigualdad de } \\
\text { ingresos entre ricos y pobres. ¿Hasta qué punto está de acuerdo o en desacuerdo } \\
\text { con esta frase? }\end{array}$ \\
\hline Pensiones & $\begin{array}{l}\text { El Estado X, más que el sector privado, debería ser el principal responsable de } \\
\text { proveer las pensiones de jubilación ¿Hasta qué punto está de acuerdo o en } \\
\text { desacuerdo con esta frase? }\end{array}$ \\
\hline Salud & $\begin{array}{l}\text { El Estado X, más que el sector privado, debería ser el principal responsable de } \\
\text { proveer los servicios de salud. ¿Hasta qué punto está de acuerdo o en desacuerdo } \\
\text { con esta frase? }\end{array}$ \\
\hline
\end{tabular}

Fuente: Elaboración propia a partir de PELA y LAPOP.

Como se observa en lo cuadro anterior, los seis temas incluidos en el análisis dan lugar a un conjunto de variables con una escala ordinal de siete puntos. A partir de dichas variables se realizará la operacionalización de la congruencia temática. Dicho proceso consta de tres pasos. Primero se debe conocer el grado de asociación entre cada par de variables. El segundo paso consiste en obtener la configuración de las relaciones entre todas las variables. Finalmente, es necesario poder medir el grado de similitud entre las estructuras de opinión de los ciudadanos y los diputados.

\section{Metodologia para la medición de la congruencia temática}

Recordemos que el análisis de la congruencia ideológica se define tanto por su enfoque teórico como por su perspectiva metodológica. En este sentido, resulta esencial especificar qué se mide y cómo se mide. La primera de estas cuestiones ya fue discutida en la sección anterior. Por su parte, la medición de la congruencia desde una perspectiva multidimensional hace necesario usar herramientas de estadística multivariante que permitan llevar a cabo dos tareas analíticas. En primer lugar, 
encontrar la estructura que subyace las relaciones entre las diferentes dimensiones (temas) estudiadas. Además, es necesario tener una manera directa de interpretación de las mismas. La segunda es que permita medir el grado de similitud entre las estructuras de los dos grupos de personas considerados. A partir de dicha medida es posible lograr una aproximación empírica al concepto de congruencia.

Para lograr dicho objetivo se hace uso de dos métodos de análisis multivariante que permiten cumplir con los requisitos mencionados: el Escalamiento Multidimensional (EMD) y el Análisis Procrustes (AP). A partir de estas técnicas es posible tener una aproximación empírica al estudio de la congruencia porque es posible representar de manera gráfica la estructura de relaciones entre los diferentes temas y comparar su grado de similitud.

El EMD es una técnica que permite la representación espacial de medidas de proximidad entre pares de objetos o estímulos (BARTHOLOMEW et al., 2008) ${ }^{12}$. Así, el EMD tiene como punto de partida una matriz de datos cuyas entradas corresponden a (dis)similitudes, $\delta_{i j}$, entre los n objetos $\Delta \in M_{n x n}{ }^{13}$. Como resultado del análisis se obtiene una matriz $X \in M_{n x m}$ donde $m$ representa el número de dimensiones de la representación espacial de las relaciones entre los $n$ estímulos (objetos). Cada elemento de esta matriz $x_{i j}$ corresponde a las coordenadas de un estímulo (i) en una dimensión (j). Por lo tanto es posible calcular la distancia entre dos elementos usando la fórmula general de Minkowski:

$$
d_{i j}=\left[\sum_{t=1}^{m}\left(x_{i t}-x_{j t}\right)^{p}\right]^{1 / p}
$$

Donde usualmente $p=2$. Estas distancias $\left(d_{i j}\right)$ se recogen en una matriz $D \in M n x n$, y el objetivo del EMD es que exista la mayor correspondencia posible entre

\footnotetext{
${ }^{12}$ En términos simples, el EMD se puede concebir como el proceso opuesto al de consultar un mapa. Generalmente, usamos un mapa para calcular la distancia entre dos puntos, en el EMD usamos la distancia entre puntos para construir un mapa.

${ }^{13}$ Una medida de similitud que es usada comúnmente en las ciencias sociales es el coeficiente de correlación bivariante. El análisis a partir de este estadístico se vuelve más complejo mientras mayor es el número de variables analizadas. El EMD permite encontrar la estructura de la relación entre un número elevado de variables de una manera bastante intuitiva y directa, generalmente en una representación bidimensional. Desde una perspectiva estadística, el EMD es una herramienta de reducción de la dimensionalidad de un conjunto de datos multivariante.
} 
las matrices $\Delta$ y $D$. La principal diferencia entre el EMD métrico y el no métrico es que en este último no se asume una relación lineal entre las disimilitudes y las distancias. En el EMD no métrico se establece una relación monótona creciente: si $\delta_{i j}<\delta_{k l} \Rightarrow d_{i j} \leq d_{\mathrm{kl}}$. Para llevar a cabo este análisis es necesario crear las disparidades $\hat{d}_{i j}$ que son obtenidas por medio de una regresión monotónica por mínimos cuadrados de las distancias sobre las disimilitudes. Estas disparidades cumplen el objetivo de tener la misma estructura ordinal de las disimilitudes. Para evaluar el ajuste de la representación se usa el stress tipo I de Kruskal:

$$
\sqrt{\frac{\sum_{i, j}\left(d_{i j}-\widehat{d}\right)^{2}}{\sum_{i, j} d_{i j}^{2}}}
$$

En este caso se ha usado el EMD no métrico porque la métrica original de los datos analizados es ordinal (RABINOWITZ, 1975; SÁNCHEZ-CARRIÓN, 1985). Además, es importante señalar que el hecho de contar como punto de partida con las similitudes correspondientes a coeficientes de correlación no significa que sea más adecuado usar un EMD métrico. De acuerdo con Weisberg y Rusk (1970), generalmente es más recomenable usar el EMD no métrico cuando las similitudes de las que partimos son correlaciones entre variables ordinales.

El análisis fue llevado a cabo a partir de las correlaciones policóricas entre los seis temas analizados. Estos coeficientes son necesarias porque, como señalan Holgado-Tello et al. (2010), cuando se analizan datos cuya escala es ordinal lo más adecuado es obtener las correlaciones policóricas de las variables. Entre otros problemas, dichos autores señalan que el usar el de correlación de Pearson con datos ordinales repercutirá en la subestimación del grado de asociación de las variables. Para estimar dichas las correlaciones policóricas, Forero et al., (2009) señalan que lo más usual es asumir que los indicadores ordinales observados $\mathrm{X}_{k}$ surgen a partir de unas variables "ficticias" (no observadas) $\mathrm{X}_{\mathrm{k}}{ }^{*}$, las cuales tienen una distribución normal. También se asume que cada variable "ficticia" genera un indicador ordinal observado a través de una operacionalización basada en los umbrales $\tau_{\mathrm{k}}$. Así, si $\mathrm{X}_{\mathrm{k}}{ }^{*}$ está por encima del umbral $\tau_{\mathrm{k}}$ y por debajo del umbral $\tau_{\mathrm{k}+1}, \mathrm{X}_{\mathrm{k}}$ tomará el valor discreto correspondiente $(0,1,2, \ldots)$. También se considera que la distribución conjunta de dos de las variables "ficticias" es normal bivariante. Así, primero se estiman los valores de 
los umbrales y luego se obtiene la correlación entre cada par de variables por medio del método de máxima verosimilitud (KOLENIKOV y ÁNGELES, 2004).

Para poder llevar a cabo una comparación entre las estructuras de las actitudes sobre los temas analizados, es importante recordar que los ejes generados en el EMD no tienen un significado intrínseco. Por lo tanto, no es viable comparar dos o más de estas configuraciones de forma directa para conocer su grado de similitud. Entonces es necesario contar con una herramienta estadística que permita evaluar qué tan similares son estas estructuras. Así, los métodos Procrustes proporcionan un estadístico y una solución gráfica que es posible interpretar como la concordancia de dos representaciones espaciales. Para llevar a cabo el análisis Procrustes es necesario contar con las coordenadas de las representaciones de dos o más grupos. A partir de los resultados de dicho análisis es posible aproximarnos al concepto de la congruencia temática, considerando que una mayor similitud significa que los representes y los representados tienen una estructura parecida con respecto a sus opiniones sobre estos temas.

Fundamentalmente, los métodos Procrustes consisten en ajustar dos o más configuraciones de puntos provenientes de las coordenadas obtenidas a partir de un análisis de reducción de dimensión (HURLEY y CATTELL, 1962). En la aplicación más básica de esta metodología se parte de dos configuraciones de puntos en un espacio r-dimensional, las cuales normalmente representan medidas tomadas sobre un mismo conjunto de objetos, y se trata de averiguar qué tan similares son las dos configuraciones. La respuesta a esta cuestión se obtiene después de aproximar estas configuraciones lo más posible por medio de tres procesos: traslación, rotación y dilatación. Finalmente, la suma de cuadrados residual que se obtiene después de aproximar las configuraciones se llama estadístico Procrustes. Para efectos del presente estudio, este indicador ofrece una medida global de la congruencia en cada uno de los países, el cual permite también comparaciones directas entre los casos estudiados.

A continuación se presenta una breve descripción formal de este método. Sean $\mathrm{X}$ y $\mathrm{Y}$ dos matrices de datos o configuraciones de puntos que se quieren comparar. A la primera de ellas se le denomina source mientras que a la segunda se le llama target. Para comenzar con la comparación entre matrices se puede asumir que éstas están centradas y estandarizadas o incluir en el modelo un factor de escalamiento. En este caso, de acuerdo con Gower y Dijksterhuis (2004), se verifica que: $\operatorname{tr}\left(\mathrm{X}^{\prime} \mathrm{X}\right)=\operatorname{tr}\left(\mathrm{Y}^{\prime} \mathrm{Y}\right)$. El objetivo, entonces, es aplicar a la configuración $\mathrm{X}$ la rotación $(\mathrm{T})$ y la dilatación por un factor $(s)$ para que difiera lo menos posible de $\mathrm{Y}$. 
Entonces, el objetivo del análisis es encontrar $\mathrm{T}$ y $s$ que minimicen la siguiente expresión:

$$
\|s \mathrm{XT}-\mathrm{Y}\|=\operatorname{tr}(s \mathrm{XT}-\mathrm{Y})^{\prime}(s \mathrm{XT}-\mathrm{Y})
$$

Así, la solución a este problema se encuentra utilizando la descomposición en valores singulares de Y’X, la cual es igual a U $\Sigma V^{\prime}$. Entonces la solución será:

$$
\mathrm{T}=\mathrm{VU}^{\prime} \text { y } \quad s=\operatorname{tr}\left(\mathrm{Y}^{\prime} \mathrm{XT}\right) /\|\mathrm{X}\|
$$

Es importante señalar que el valor mínimo de $\|s \mathrm{XT}-\mathrm{Y}\|$ se denomina estadístico Procrustes - que puede interpretarse como una medida de distancia/cercanía entre dos matrices de datos - el cual tiene un rango teórico que va de 0 a 1 . Los valores cercanos al mínimo denotan una fuerte similitud entre las configuraciones de puntos. Por otro lado, los valores cercanos al máximo significan que las matrices son muy diferentes. En el presente trabajo, el análisis Procrustes se llevó a cabo a partir de las coordenadas correspondientes a las configuraciones de puntos obtenidas por medio del análisis EMD. En todos los casos las coordenadas de la configuración obtenida para los ciudadanos se usaron como matriz target.

\section{Niveles de congruencia temática en los paises latinoamericanos}

Debido a la gran cantidad de información obtenida al realizar el análisis empírico, donde fueron generados 45 gráficos (tres para cada país), sólo serán discutidos en detalle dos casos. Para el resto de países se presentarán los estadísticos Procrustes ${ }^{14}$. En primer lugar se encuentra Brasil, el país que obtuvo el menor estadístico Procrustes por lo que representa el caso con más alta congruencia temática.

La Tabla 1 muestra las correlaciones policóricas entre las seis variables para los dos grupos. Debajo de la diagonal se encuentran las correspondientes a los ciudadanos y encima de la diagonal las de los diputados. Se puede observar en el primer grupo que todas las variables, excepto "empresas", están altamente correlacionadas entre sí. Este es un patrón que se encontró en varios países. Para los diputados, la estructura de correlaciones muestra valores menores y una configuración ligeramente diferente. Al contrario de los ciudadanos, la variable "pensiones" tiene

\footnotetext{
${ }^{14}$ Todos los gráficos Procrustes se encuentran en el apéndice.
} 
una mayor correlación con "empresas" que con "bienestar" y "empleo". Aunque esta información es útil, a partir de esta tabla es difícil tener una idea de la estructura de interrelaciones que tienen estos temas para los grupos de personas analizados.

Tabla 1 - Correlaciones policóricas Brasil

\begin{tabular}{l|c|c|c|c|c|c}
\hline & Empresas & Bienestar & Empleo & Desigualdad & Pensiones & Salud \\
\hline Empresas & 1 & 0.338 & 0.326 & 0.142 & 0.218 & 0.294 \\
\hline Bienestar & 0.419 & 1 & 0.617 & 0.597 & 0.213 & 0.560 \\
\hline Empleo & 0.364 & 0.650 & 1 & 0.407 & 0.170 & 0.504 \\
\hline Desigualdad & 0.275 & 0.589 & 0.609 & 1 & 0.383 & 0.635 \\
\hline Pensiones & 0.303 & 0.523 & 0.556 & 0.596 & 1 & 0.450 \\
\hline Salud & 0.234 & 0.548 & 0.590 & 0.644 & 0.728 & 1 \\
\hline
\end{tabular}

Fuente: Elaboración propia.

Como se discutió anteriormente, por medio del análisis EMD es posible tener una representación accesible en dos dimensiones de esta estructura de relaciones multidimensional. En el siguiente gráfico se presentan las configuraciones obtenidas a través del EMD a partir de las correlaciones mostradas en la Tabla 2. A primera vista parece que las estructuras de opinión de los ciudadanos y los diputados presentan diferencias relevantes. Los ítems, excepto "empresas", se encuentran en lados opuestos del eje vertical. Sin embargo, es importante recordar que las configuraciones del EMD representan distancias relativas entre los ítems analizados. Así que no hay una manera directa de comparar las posiciones de los temas en las configuraciones de dos grupos distintos. También es posible observar que, tanto para los ciudadanos como para los diputados, la variable "empresa" no está fuertemente asociada con las demás ${ }^{15}$.

${ }^{15}$ Uno de los objetivos del análisis EMD es buscar una interpretación sustantiva a los ejes que marcan las posiciones de los ítems. Algunos autores sugieren hacerlo por medio de alguna variable externa que dé sentido a las dimensiones (RABINOWITZ, 1975). En este caso no se llevó a cabo dicha interpretación porque el objetivo es medir el grado de similitud entre las configuraciones de ciudadanos y diputados. 
Gráfico 1- Representaciones EMD para Brasil

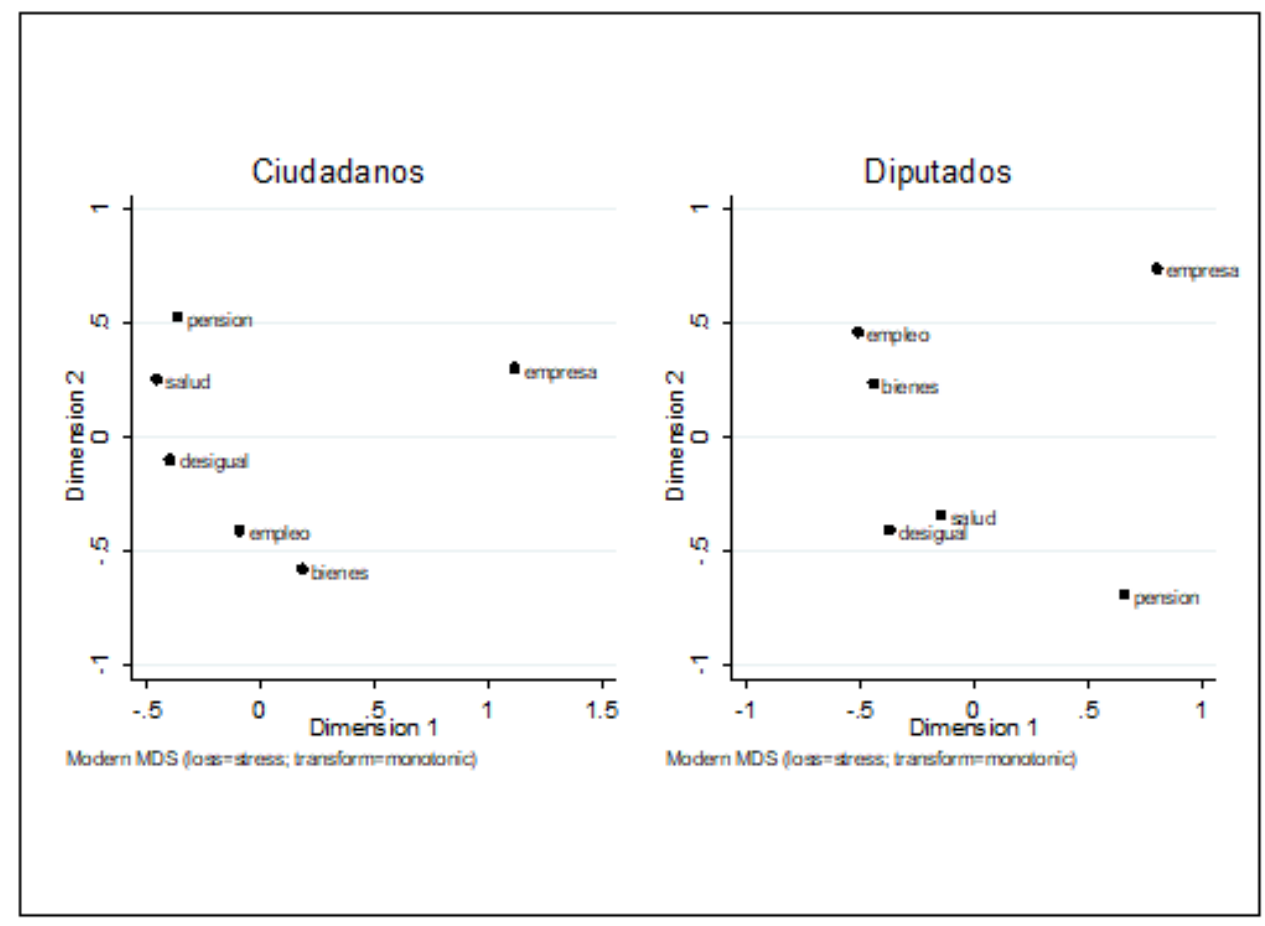

Fuente: Elaboración propia.

Al observar detenidamente las dos configuraciones del gráfico anterior es posible percibir que si se rotan los puntos alrededor del eje horizontal las configuraciones se vuelven muy similares. Como se discutió anteriormente, el AP es una herramienta estadística que permite llevar a cabo este tipo de transformaciones de manera sistemática para comparar dos o más configuraciones. El resultado de dicho análisis se presenta en el Gráfico 2. Las dos estructuras son casi idénticas. También es posible observar que las distancias ítem por ítem son muy pequeñas. Esto se ve reflejado en el valor del estadístico Procrustes 0.1248 (donde 0 es el mínimo teórico posible y 1 el máximo). 
Gráfico 2- Representación Procrustes para Brasil

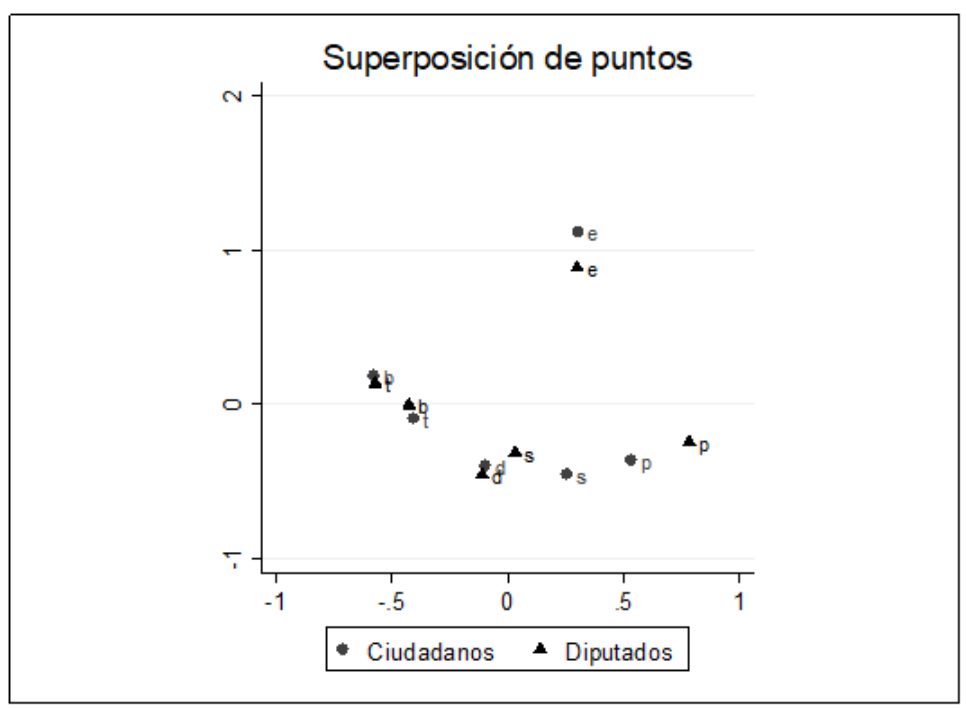

Fuente: Elaboración propia.

Para llevar a cabo el contraste, se discute a continuación el caso de Nicaragua que es el país que obtuvo el mayor valor del estadístico Procrustes. Como se ha mencionado anteriormente, esto se traduce en menor congruencia. En la Tabla 2 se encuentran las correlaciones para las seis variables analizadas en el grupo de ciudadanos y en el de diputados ${ }^{16}$.

Tabela 2 - Correlaciones policóricas de Nicaragua

\begin{tabular}{l|c|c|c|c|c|c}
\hline & Empresas & Bienestar & Empleo & Desigualdad & Pensiones & Salud \\
\hline Empresas & 1 & -0.021 & 0.686 & 0.059 & 0.230 & -0.144 \\
\hline Bienestar & 0.177 & 1 & 0.250 & 0.409 & 0.157 & 0.407 \\
\hline Empleo & 0.077 & 0.669 & 1 & -0.053 & 0.188 & -0.005 \\
\hline Desigualdad & 0.079 & 0.489 & 0.562 & 1 & 0.170 & 0.518 \\
\hline Pensiones & 0.028 & 0.583 & 0.675 & 0.540 & 1 & 0.285 \\
\hline Salud & -0.027 & 0.607 & 0.719 & 0.575 & 0.791 & 1
\end{tabular}

Fuente: Elaboración propia.

${ }^{16}$ Al igual que en el caso de Brasil, las correlaciones de los ciudadanos se encuentran debajo de la diagonal y las de los diputados encima. 
En este caso los patrones de correlaciones son bastante diferentes. Por un lado, los ciudadanos muestran una estructura similar a la observada en otros países. Las correlaciones entre todos los temas son relativamente altas, excepto en "empresas". En el grupo de diputados, el patrón tiene importantes diferencias con el de los ciudadanos. Por ejemplo, "empleo" y "empresas" se encuentran altamente correlacionadas y "pensiones" tiene su máxima correlación con ésta última. Además, entre los representantes políticos se presentan varias correlaciones negativas. Este es el único caso que muestra dicho patrón. Para tener una perspectiva de la estructura multivariante de estas relaciones se presenta el siguiente gráfico, correspondiente al análisis EMD.

Gráfico 3 - Representaciones EMD para Nicaragua

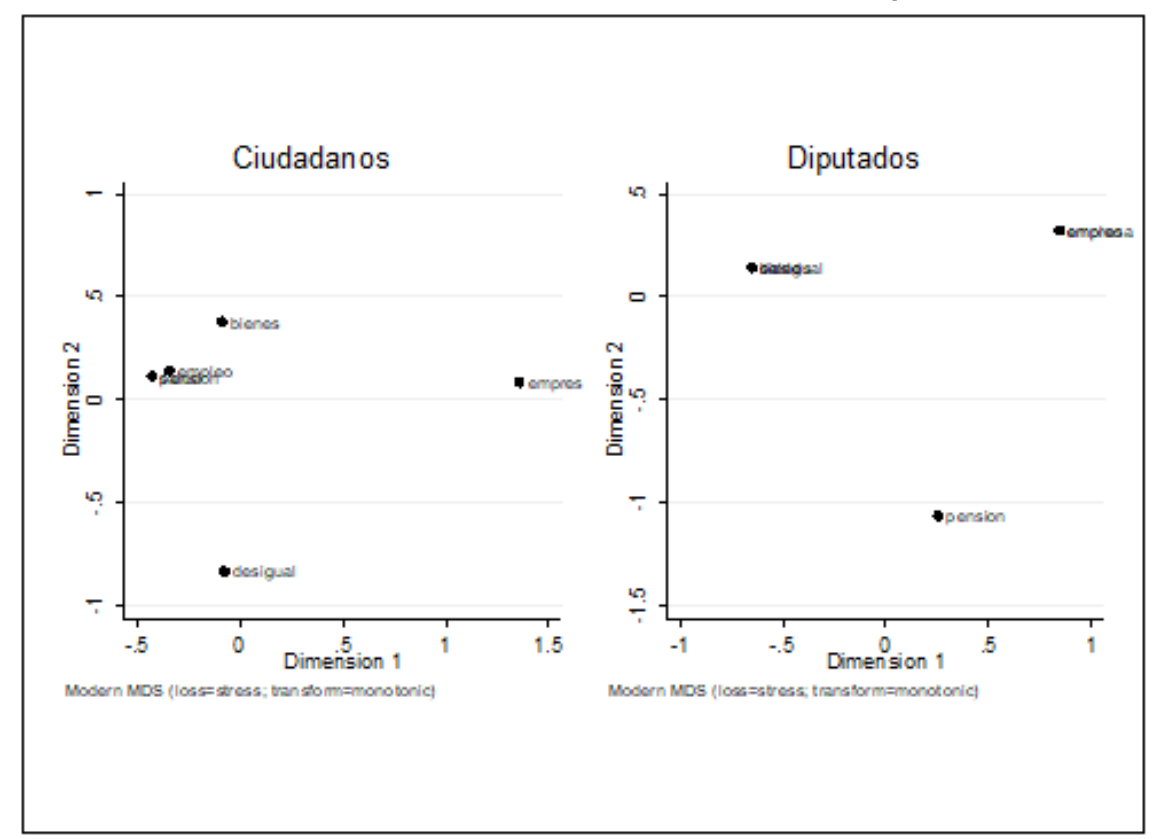

Fuente: Elaboración propia.

En el Gráfico 3 se puede observar que las configuraciones tienen diferencias significativas. Para los ciudadanos la función del Estado como principal responsable del combate a la desigualdad no parece estar relacionada con los demás temas. Además, al igual que en la mayoría de los países analizados, la variable "empresas" tampoco está asociada con los otros ítems. En cuanto a los diputados, los seis temas 
parecen estar estructurados en dos bloques y un tema "pensiones" casi equidistante a estos. Al igual que en el caso anterior, se llevó a cabo el AP para conocer el grado de similitud entre estas estructuras.

Gráfico 4 - Representación Procrustes para Nicaragua

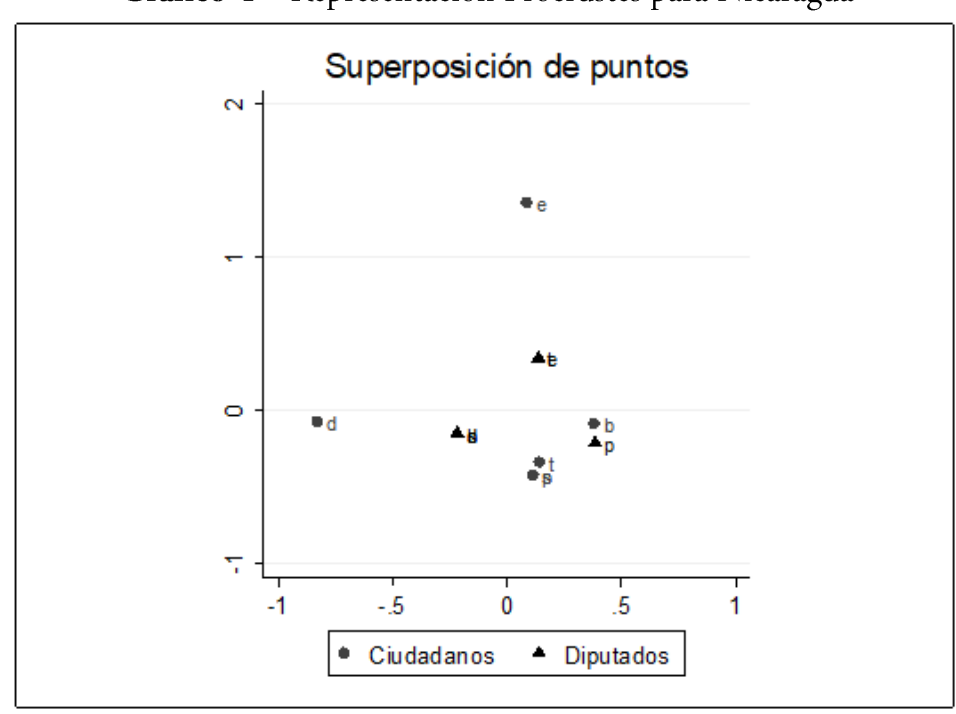

Fuente: Elaboración propia.

En el gráfico anterior se observa que las distancias ítem por ítem son claramente más grandes que en el caso de Brasil. Las mayores distancias se presentan en las variables "empresas", "empleo" y "desigualdad". Esta situación se ve reflejada en el alto valor del estadístico Procrustes (0.791), correspondiente al nivel más bajo de concordancia de las estructuras multivariantes.

Con el objetivo de tener un panorama general de la congruencia temática en América Latina, se presenta un gráfico con los estadísticos Procrustes para todos los casos analizados. Estos están ordenados de menor puntuación (mayor concordancia) a mayor puntuación (menor concordancia). Los casos correspondientes a los valores mínimo y máximo han sido discutidos en los párrafos anteriores. 


\section{Gráfico 5 - Estadístico Procrustes para los Países de América Latina}

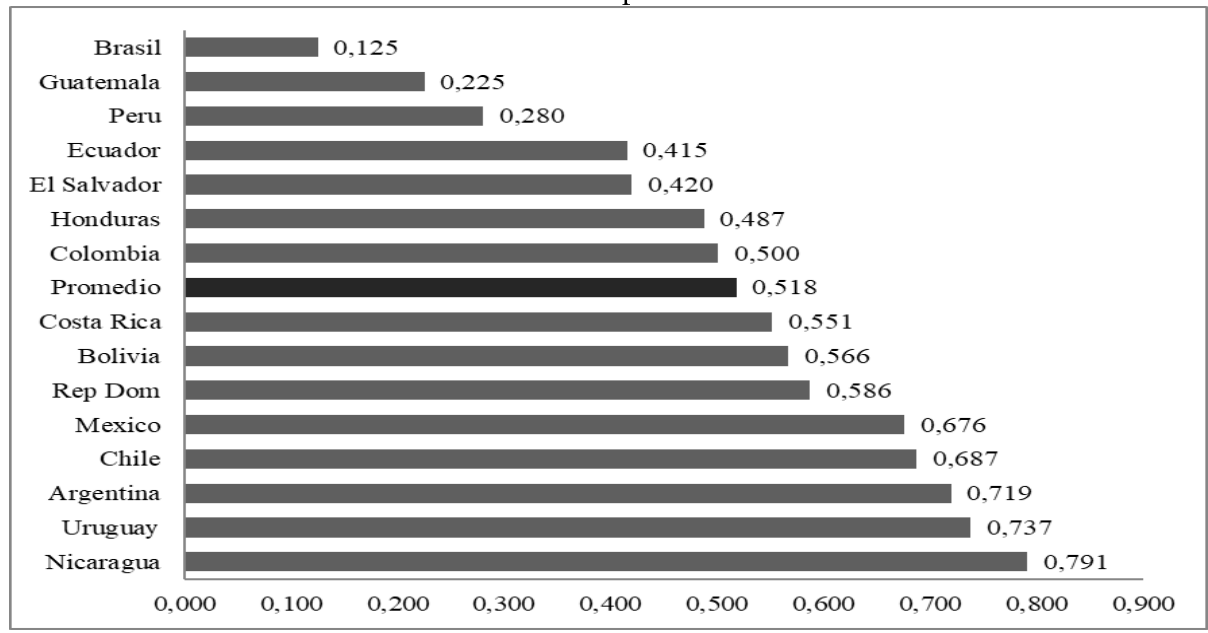

Fuente: Elaboración propia.

En términos generales, es posible observar que no existe un patrón claro respecto al tipo de países que tienen niveles bajos (altos) de concordancia en la forma como están estructurados los temas de la intervención estatal. En el Gráfico 5 podemos encontrar países por debajo de la media con sistemas de partidos que se consideran poco institucionalizados, por ejemplo Brasil y Perú. Por otro lado, entre los países con mayores valores de este indicador (menor congruencia) hay algunos con sistemas de partidos consolidados como Uruguay y Chile. Así, para poder explicar el grado de congruencia temática de los países latinoamericanos será necesario considerar factores que tengan que ver con la oferta partidista que reciben los electores. Un ejemplo de dichos elementos es el número de partidos con representación legislativa, donde un número alto de partidos puede generar un mayor rango de opiniones sobre los temas estudiados lo cual repercutiría en que las opiniones de los ciudadanos se verían mejor reflejadas en el parlamento. Otro posible factor es el nivel de riqueza de los países. Al parecer los más ricos tienden a tener menores niveles de congruencia sobre estos temas. Quizás las élites políticas de los países que tienen mayor nivel de desarrollo económico conciben las relaciones entre estos temas desde una perspectiva centrada en criterios técnicos. Por otro lado, es posible que los ciudadanos sigan conceptualizando dichas relaciones con una visión más "paternalista" del Estado.

A partir de los hallazgos anteriores, es necesario conocer cuáles son los temas que generan mayor discrepancia entre ciudadanos y diputados. Las representaciones 
espaciales obtenidas a partir del AP (ver Apéndice) señalan que las mayores distancias en la posición relativa de los ítems corresponden a la variable "empresas". En otras palabras, la principal fuente de divergencia en las posiciones de los ciudadanos y los diputados es la opinión hacia la propiedad por parte del Estado de las principales empresas del país. La diferencia en la relación entre la variable "empresas" y el resto de variables tiene un efecto importante en el nivel de congruencia de las actitudes hacia la intervención estatal de los ciudadanos y diputados de la región. El análisis se repitió usando sólo las otras cinco variables y los resultados variaron significativamente, la correlación entre los estadísticos Procrustes en ambos escenarios es muy baja (0.13). Con respecto al tema cuya relación con los demás fue más similar para diputados y ciudadanos, en ningún caso la mayor distancia correspondió a "salud". Este hallazgo puede ser interpretado como que tanto ciudadanos como diputados tienen una postura similar sobre la provisión de servicios de salud por parte del Estado, relativa a los otros temas.

Los resultados anteriores evidencian la ventaja de llevar a cabo el análisis de la congruencia temática por pasos, dado su carácter multidimensional. De esta manera se pueden identificar aquellos ítems que tienen mayor divergencia entre los grupos. También es posible evaluar los cambios longitudinales en la estructura de relaciones entre los temas dentro de cada país.

\section{Conclusiones}

En el presente artículo se ha propuesto una contribución al estudio de la congruencia en América Latina desde un punto de vista empírico. Por medio de dos herramientas de estadísticas, el Escalamiento Multidimensional y el Análisis Procrustes, ha sido posible estimar el grado de congruencia temática en quince países de esta región. Así, esta aportación es útil porque aprovecha las ventajas del análisis multivariante para poder aproximarse al estudio de un tema con varias dimensiones. De esta manera ha sido posible analizar un fenómeno complejo por medio de mediciones objetivas que han permitido llevar a cabo comparaciones entre diferentes países. Además, este estudio ha evitado los problemas que se derivan de analizar la congruencia entre representantes y representados a través de un indicador unidimensional como es la ideología.

Esta es un área que está recibiendo cada vez más atención en los estudios sobre representación política en América Latina. Sin embargo, la mayoría de los trabajos publicados se centran en un solo país. Dicha estrategia aporta un nivel de profundidad muy interesante pero no permite tener una perspectiva más amplia sobre 
el fenómeno. Por su parte, un estudio comparativo provee la oportunidad de tener mayor grado de generalidad en la comprensión de este fenómeno. El reto de esta aproximación es tener una operacionalización del objeto de estudio con los niveles de validez y fiabilidad necesarios para alcanzar conclusiones relevantes.

Aquí se ha propuesto una estrategia de medición de la congruencia temática usando herramientas de estadística multivariante. De esta manera se ha tomado en cuenta en la operacionalización de la congruencia el carácter multidimensional de dicho fenómeno. Para el análisis se ha usado evidencia empírica correspondiente a quince países Latinoamericanos, sobre seis cuestiones relativas a la intervención del Estado en la economía. Estos son temas que han tenido relevancia en la discusión política de estas sociedades durante décadas. Además, desde un punto de vista empírico, fueron operacionalizados a partir de preguntas donde ambos grupos de personas contestaron a preguntas con idéntica redacción y opciones de respuesta.

Los resultados del análisis han mostrado que los ciudadanos y los diputados de esta región comparten su perspectiva sobre algunos temas y no sobre otros. El caso más claro es el de la propiedad por parte del Estado de las principales empresas del país. La relación de este tema con los demás genera discrepancia en la conceptualización global que tienen sobre la intervención Estatal los dos grupos. En cuanto a los niveles de congruencia, medidos a través del estadístico Procrustes, el panorama es llamativo. Existe una variación importante, los países con mayor concordancia en sus estructuras multivariantes son Brasil y Guatemala, mientras que los que tienen los niveles más bajos son Uruguay y Nicaragua. No parece haber una razón clara que explique estos resultados.

La agenda de investigación sobre este fenómeno permanece abierta y es necesario seguir profundizando en su estudio. El siguiente paso será encontrar los factores que explican los diferentes de congruencia temática que presentan estos países. A partir de ahí será posible contrastar estos hallazgos con otras regiones del mundo, tanto aquellas que han sido ampliamente estudiadas como las que todavía no han recibido suficiente atención.

Juan Antonio Rodríguez-Zepeda é Doutorando da Universidade de Salamanda. E Professor Associado de Ciência Politica na Universidade de Burgos. Email: jarz@usal.es. 


\section{Referências}

ACHEN, Chris. Measuring Representation: Perils of the correlation coefficient. American Journal of Political Science, v. 21, n. 4, p. 805-815, 1977.

ACHEN, Chris. Measuring Representation. American Journal of Political Science, v. 22, n. 3, p. 475510, 1978.

BARTHOLOMEW, David J.; STEELE, Fiona; GALBRAITH, Jane; MOUSTAKI, Irini. Analysis of Multivariate Social Science Data. CRC Press, ed. 2, 2008.

BLAIS André; BODET, Marc André. Does Proportional Representation Foster Closer Congruence between Citizens and Policymakers? Comparative Political Studies, v. 39, p. 1243-1263, 2006.

BUDGE, Ian; KLINGEMANN, Hans-Dieter; VOLKENS, Andrea; BARA, Judith; TANENBAUM, Eric (Eds). Mapping policy preferences: estimates for parties, electors, and governments 1945-1998. Oxford: Oxford University Press, 2001.

BURSTEIN, Paul. The impact of Public Opinion on Public Policy: A review and Agenda. Political Research Quarterly, v. 56, n. 1, p. 29-40, 2003.

CASTLES, Francis; MAIR, Peter. Left-Right Political Scales: Some 'Expert' Judgments. European Journal of Political Research, v. 12, p. 73-88, 1984.

CONVERSE, Philip; PIERCE, Roy. Political Representation in France. Cambridge, Mass.: Harvard University Press, 1986.

DALTON, Russell. Political Parties and Political Representation: Party Supporters and Party Elites in Nine Nations. Comparative Political Studies, v. 18, p. 267-299, 1985.

FLORES-MACÍAS, Gustavo. After Neoliberalism?: The Left and Economic Reforms in Latin America. Oxford: University Press, 2012.

GOLDER, Matthew; STRAMSKI, Jacek. Ideological Congruence and Electoral Institutions. American Journal of Political Science, v. 54, n. 1, p. 90-106, 2010.

GOWER John; DIJKSTERHUIS, Garmt. Procrustes Problems. Oxford: Statistical Science Series, 2004.

GRAMACHO, Wladimir; LLAMAZARES, Iván. Ideología y preferencias de intervención económica estatal entre los diputados latinoamericanos. Documentos CIDOB. Serie América Latina, n. 18, 2007.

HERRERA, Cheryl Lyn; HERRERA Richard; SMITH, Eric. Public Opinion and Congressional Representation. Public Opinion Quarterly, v. 56, p. 185-205, 1992.

HOLGADO-TEllo, Francisco Pablo; CHACÓN-MOSCOSO, Salvador; BARBERO-GARCÍA, Isabel; VILA-ABAD, Enrique. Polychoric versus Pearson correlations in exploratory and confirmatory factor analysis of ordinal variables. Quality \& Quantity, v. 44, n. 1, p. 153-166, 2010.

HURLEY, John R.; CATTELL, Raymond B. The Procrustes program: Producing direct rotation to test a hypothesized factor structure. Behavioral Science, v. 7, p. 258-262, 1962.

KOLENIKOV, Stanislav; ANGELES, Gustavo. The Use of Discrete Data in Principal Component Analysis with Applications to Socio-Economic Indices. CPC/MEASURE Working paper, p. 4-85, 2004

LUNA, Juan Pablo. Segmented Representation: Political Party Strategies in Unequal Democracies. New York: Oxford University Press, 2014.

LUNA, Juan Pablo; ZECHMEISTER, Elizabeth. Representation in Latin America: A Study of EliteMass Congruence in 9 Countries. Comparative Political Studies, v. 38, n. 4, p. 388-416, 2005. 
LUPU, Noam; SELIOS Lucia; WARNER, Zach. A New Measure of Congruence: The Earth Mover's Distance. Political Analysis, v. 25, p. 95-113, 2017.

MILLER, Warren E.; STOKES, Donald. Constituency Influence in Congress. American Political Science Review, v. 57, p. 45-56, 1963.

OTERO-FELIPE, Patricia. Congruencia ideológica en América Latina desde una perspectiva dinámica: ¿Continuidad o cambio? In: RUIZ-RODRÍGUEZ, Leticia. (Org.) Percepciones y actores de la representación política en América Latina. Barcelona: Huygens, 2017. p. 81-106.

OTERO-FELIPE, Patricia; RODRÍGUEZ-ZEPEDA, Juan Antonio. Measuring Political Representation in Latin America: A Study of the Ideological Congruence between Parties and Voters, Conferencia Anual American Political Science Association (APSA), Washington, p. 1-4 septiembre, 2010. Disponível em: <http://papers.ssrn.com/sol3/papers.cfm?abstract_id=1642532>. Acesso em 12 set. 2017.

OTERO-FELIPE, Patricia; RODRÍGUEZ-ZEPEDA, Juan Antonio. Vínculos ideológicos y rendimiento electoral en América Latina. Política y Gobierno, v. 21, n. 1, p. 159-200, 2014.

POWELL, Bingham G. Political representation in comparative politics. Annual Review of Political Science, v. 7, p. 273-296, 2004.

RABINOWITZ, George B. An Introduction to Nonmetric Multidimensional Scaling. American Journal of Political Science, v. 19, p. 343-390, 1975.

SAIEGH, Sebastian. Using Joint Scaling Methods to Study Ideology and Representation: Evidence from Latin America. Political Analysis, v. 23, n. 3, p. 363-384, 2015.

SÁNCHEZ CARRIÓN, Juan Javier. Introducción al Análisis Multidimensional No-Métrico. Revista Española de Investigaciones Sociológicas, v. 29, p. 187-126, 1985.

STOKES, Susan. Mandates and Democracy: Neoliberalism by Surprise in Latin America. Cambridge: University Press, 2001.

THOMASSEN, Jacques; SCHMITT, Hermann. Introduction: Political Representation and Legitimacy in the European Union. In: SCHMITT, Hermann; THOMASSEN, Jacques (Org.). Political representation and legitimacy in the European Union. Oxford, UK: Oxford University Press, 1999.

WEISBERG, Herbert. F.; RUSK, Jerrold G. Dimensions of Candidate Evaluation. American Political Science Review, v. 64, p. 1167-1185, 1970.

WEISSBERG, Robert. Collective vs. Dyadic Representation in Congress. American Political Science Review, v. 72, p. 535-547, 1978.

ZECHMEISTER, Elizabeth. What's left and who's right? A Q-method study of individual and contextual influences on the meaning of ideological labels. Political Behavior, v. 28, n. 2, p. 151$173,2006$.

Texto recebido em 20 de outubro de 2017. Aprovado em 30 de outubro de 2017. 
54 I Juan Antonio Rodríguez-Zepeda

\section{Apéndice}

Gráfico A1 - Casos con alto nivel de congruencia

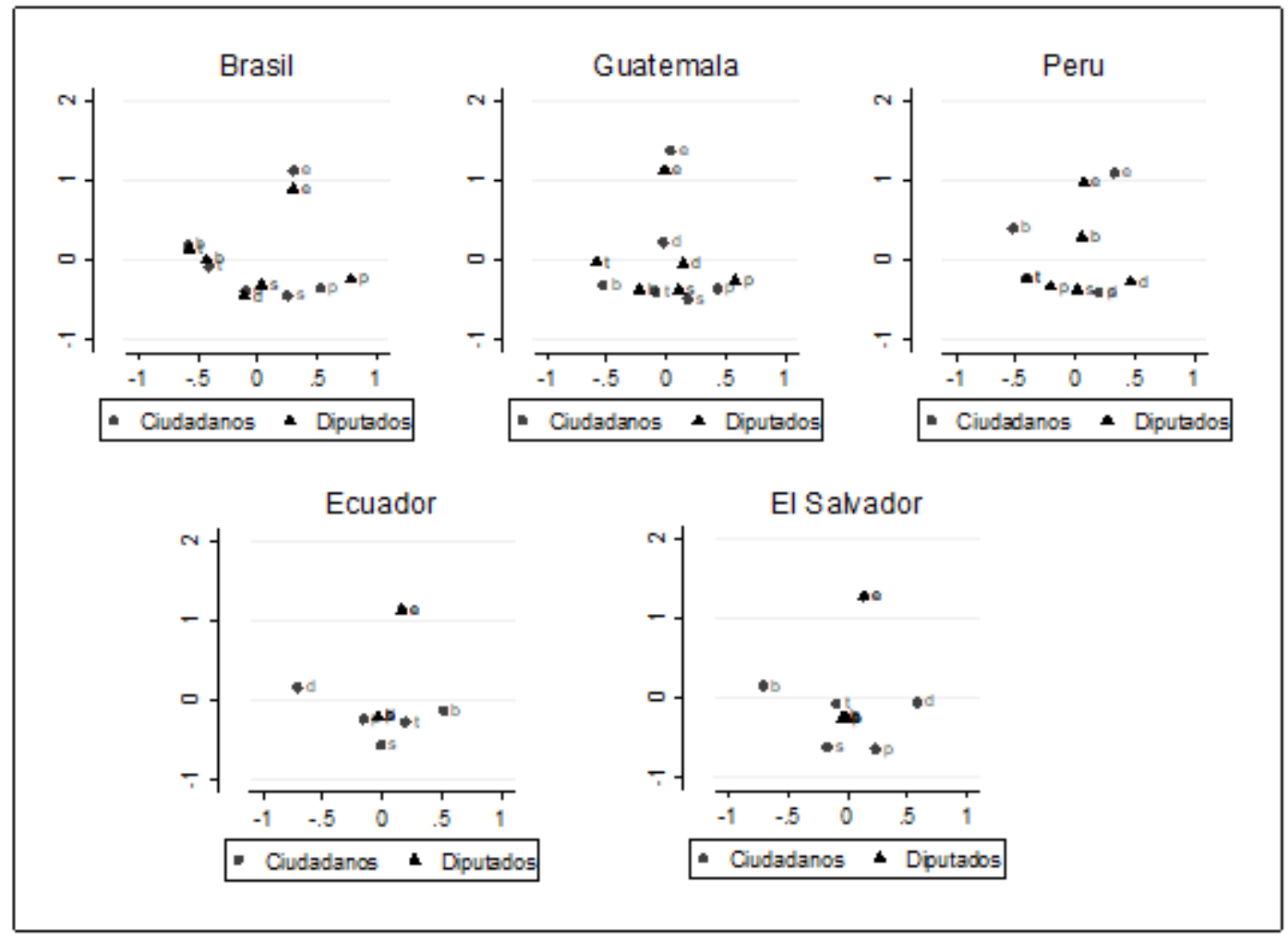

Fuente: Elaboración propia. 
Gráfico A2 - Casos con nivel medio de congruencia

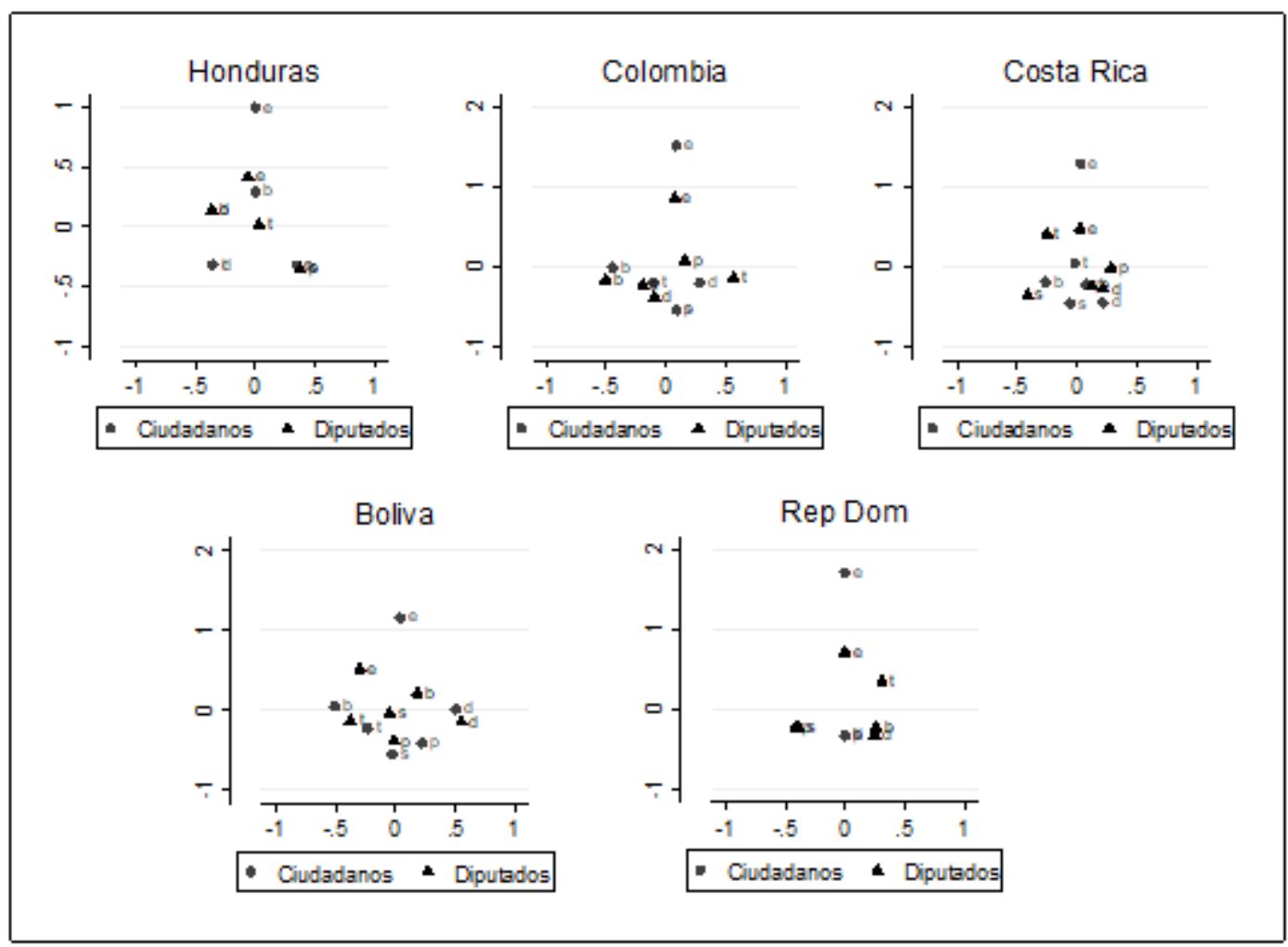

Fuente: Elaboración propia. 
Gráfico A3 - Casos con nivel bajo de congruencia

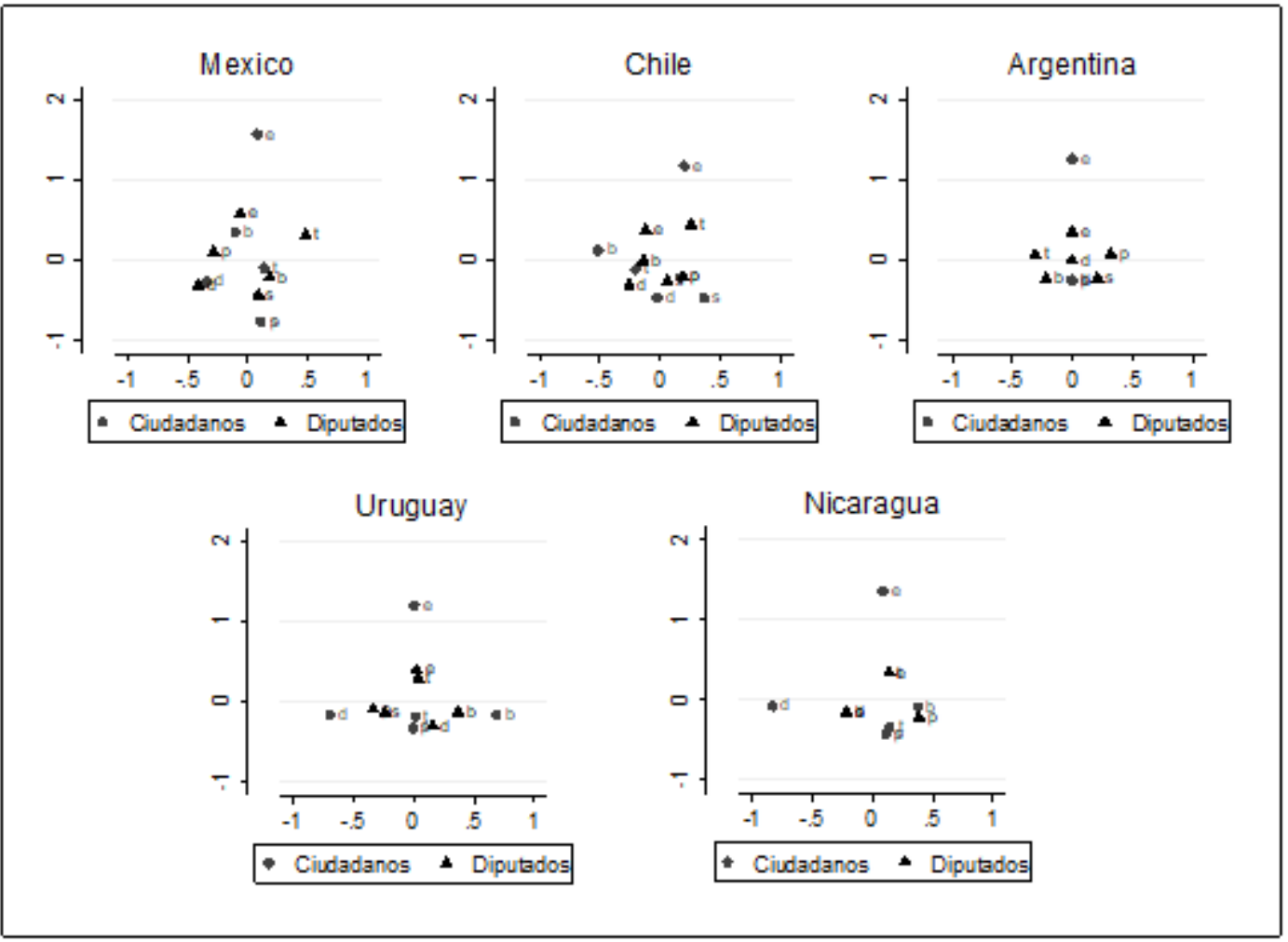

Fuente: Elaboración propia. 